\title{
Estructura y diversidad en selva inundable al centro y sur de Calakmul
}

\section{Structure and diversity in floodable jungle in central and southern Calakmul}

\author{
Websters Chiquini-Heredia ${ }^{1}$, Ligia Esparza-Olguín ${ }^{1 *}$, Yuri Peña-Ramírez ${ }^{1}$, Aixchel Maya-Martínez ${ }^{2}$, Eduardo \\ Martínez-Romero ${ }^{3}$ \\ ${ }^{1}$ Departamento Conservación de la Biodiversidad, El Colegio de la Frontera Sur-Unidad Campeche. Av. Rancho Polígono 2-A, \\ Ciudad industrial, CP. 24500. Lerma, Campeche, México \\ ${ }^{2}$ Instituto Nacional de Investigaciones Forestales, Agrícolas y Pecuarias, Centro Experimental-Edzna \\ ${ }^{3}$ Investigación y Soluciones Socioambientales AC \\ ${ }^{*}$ Autor de correspondencia: esparzaligia@gmail.com
}

Artículo científico recibido: 07 de agosto de 2015 aceptado: 07 de febrero de 2017

RESUMEN. Se establecieron nueve sitios en diferentes comunidades del centro y sur del municipio de Calakmul, con la finalidad de describir la estructura y composición de especies arbóreas en la selva inundable (SI), conocida como bajos inundables o vegetación de $A k^{\prime}$ ‘alches. Se censaron 2212 individuos, 112 especies y 36 familias. El área basal varío entre 5.6 y $18.2 \mathrm{~m}^{2} \mathrm{ha}^{-1}$, las especies con mayor valor de importancia fueron Coccoloba reflexiflora, Gymnanthes lucida, Haematoxylum campechianum, Manilkara zapota, Matayba oppositifolia, Metopium brownei y Sebastiania adenophora. La mayor similitud se presentó entre los sitios al centro del municipio, la diversidad en los sitios Carlos A. Madrazo, El Carmen II, Josefa Ortiz de Domínguez, Centauro del Norte, Ley de Fomento Agropecuario, Narciso Mendoza, Nuevo Conhuás, Unidad y Trabajo, no mostraron diferencias significativas $(p<0.05)$. La estructura horizontal de la vegetación fue similar en todos los sitios, agrupando la mayor cantidad de individuos, más del $50 \%$, en las categorías diamétricas de 2.5 a $8.4 \mathrm{~cm}$. La estructura vertical presentó un comportamiento semejante entre sitios, concentrándose el $50 \%$ de los individuos entre los 5 y $10 \mathrm{~m}$. La SI alberga especies que son prioritarias para la conservación, tanto en programas nacionales como internacionales, y dada su importancia por aporte de agua y alimento a la fauna de la región se considera que son áreas en las cuales se deben implementar programas de conservación.

Palabras clave: Diversidad estructural, valor de importancia relativa, especies leñosas dominantes

ABSTRACT. Nine sites were set in different communities in central and southern Calakmul Municipality in order to describe the structure and composition of arboreous species in the floodable jungle (SI), also known as floodable lowlands or vegetation of Ak'alches. 2212 individuals, 112 species and 36 families were counted in the census. The baseline area varied between 5.6 and $18.2 \mathrm{~m}^{2} \mathrm{ha}^{-1}$, and species with greater importance were Coccoloba reflexiflora, Gymnanthes lucida, Haematoxylum campechianum, Manilkara zapota, Matayba oppositifolia, Metopium brownei and Sebastiania adenophora. The greater similarity was between the sites in the central part of the municipality; the diversity of the Carlos A. Madrazo, El Carmen II, Josefa Ortiz de Domínguez, Centauro del Norte, Ley de Fomento Agropecuario, Narciso Mendoza, Nuevo Conhuás, Unidad y Trabajo sites did not show significant differences $(p<$ $0.05)$. The horizontal structure of vegetation was similar in all sites. The greater amount of individuals plus $50 \%$ were grouped in the categories between 2.5 and $8.4 \mathrm{~cm}$. The vertical structure showed similar behavior between sites; $50 \%$ of individuals was concentrated between 5 an $10 \mathrm{~m}$. The SI has species whose conservation has priority both in national and international programs. In view of their importance in supply of water and food for the fauna of the region, it is considered that they are areas in which conservation programs should be implemented in.

Key words: Structural diversity, relative importance value, dominant ligneous species 


\section{INTRODUCCIÓN}

El concepto de selva inundable (SI) hace referencia a la vegetación que se desarrolla sobre suelos anegados temporal o permanentemente, donde la composición de especies y la estructura de las comunidades varía con la frecuencia y los ciclos de inundación (Palacio-Aponte et al. 2002). Lo habitual es encontrar este tipo de vegetación en los bordes u orillas de algún cuerpo de agua que al desbordarse o aumentar su cauce inunda las áreas en las que se desarrolla (Marks et al. 2014). La SI se desarrolla en planicies que se inundan en alguna época del año, debido a la acumulación del agua de lluvia y permeabilidad del suelo, conociéndose en el sureste de México como vegetación de bajos o Ak'alches (Tun-Dzul et al. 2008, Villalobos-Zapata y Mendoza-Vega 2010). La SI ubicada en el municipio de Calakmul, es una matriz con condiciones ambientales peculiares con predominio de selva subperennifolia (Galindo-Leal 1999, Palacio-Aponte et al. 2002).

La composición y estructura de la vegetación en el municipio de Calakmul se relaciona con los gradientes topográficos, la dinámica de nutrientes, las características del suelo, la geología y las actividades humanas (Martínez y Galindo-Leal 2002). La SI se encuentra situada en planicies acumulativas rodeada de lomeríos en forma de cuenca, los procesos aluviales de sedimentación permiten la acumulación de materiales orgánicos e inorgánicos que inhiben la infiltración y permiten la acumulación de agua en el suelo (Palacio-Aponte et al. 2002). Este ecosistema es fuente de agua para los habitantes de las localidades, hábitat de especies epífitas y refugio o área de anidación y reproducción de la fauna (Villalobos-Zapata y Mendoza-Vega 2010). Desafortunadamente, como el resto de las selvas de la región, la SI está inmersa en procesos de cambio de uso de suelo que originan deforestación y fragmentación (García-Licona et al. 2014). Al respecto, Martínez y Galindo-Leal (2002) mencionan que la SI de Calakmul ha sido objeto de explotación selectiva, lo que posiblemente favoreció cambios en la composición de especies. A pesar de su impor- tancia ecológica y de los problemas que enfrenta la $\mathrm{SI}$ en este municipio, se tienen pocos estudios que describan su diversidad y estructura florística (Tun-Dzul et al. 2008). Por lo anterior el objetivo del trabajo fue describir la diversidad y la estructura de especies arbóreas en la SI al sur y centro del municipio de Calakmul.

\section{MATERIALES Y MÉTODOS}

\section{Área de estudio}

El estudio se realizó en nueve localidades del municipio de Calakmul, que se encuentran ubicadas entre los $19^{\circ} 12^{\prime} 00^{\prime \prime}$ y $17^{\circ} 48^{\prime} 39^{\prime \prime} \mathrm{LN}$ y, 89 $09^{\circ} 04^{\prime \prime}$ y $90^{\circ} 28^{\prime} 05^{\prime \prime}$ de LO (Figura 1). Las cuales fueron: Centauro del Norte (CN), El Carmen II (EC), Narciso Mendoza (NM) y Nuevo Conhuás (NC) al centro del municipio; Arroyo Negro (AN), Carlos A. Madrazo (CM), Josefa Ortiz Domínguez (JOD), Ley de Fomento Agropecuario (LFA) y Unidad y Trabajo (UT) al sur del municipio. Los sitios se caracterizan por tener más de 40 años sin usarse para actividades agropecuarias o forestales, sin evidencias de actividad humana reciente con selva baja subperennifolia, suelo tipo $A k^{\prime}$ alche. Posiblemente, al igual que el resto de las selvas de la región de Calakmul, hayan estado expuestas a extracción de madera y de chicle entre 1930 y 1970 (Kepleis 2004). El clima del municipio es cálido subhúmedo (García 1988). La temporada de lluvias comprende de junio a noviembre con precipitación media anual de $1076.2 \mathrm{~mm}$, y temperatura promedio de $24.6{ }^{\circ} \mathrm{C}$ (Martínez y Galindo-Leal 2002). La altitud en el centro del municipio es de 200 a $250 \mathrm{msnm}$ con declives menores al $10 \%$, al sur pueden encontrarse altitudes menores a los 100 msnm (INEGI 2014).

\section{Diseño de muestreo}

La selección de las comunidades para la instalación de las parcelas se realizó al azar, tomando en cuenta todas las comunidades al centro y sur de Calakmul en las cuales se tiene registró de la SI, mientras que la selección de parcelas en cada comunidad se realizó de forma sistemática. Se establecieron sitios de muestreo circulares de 1000 


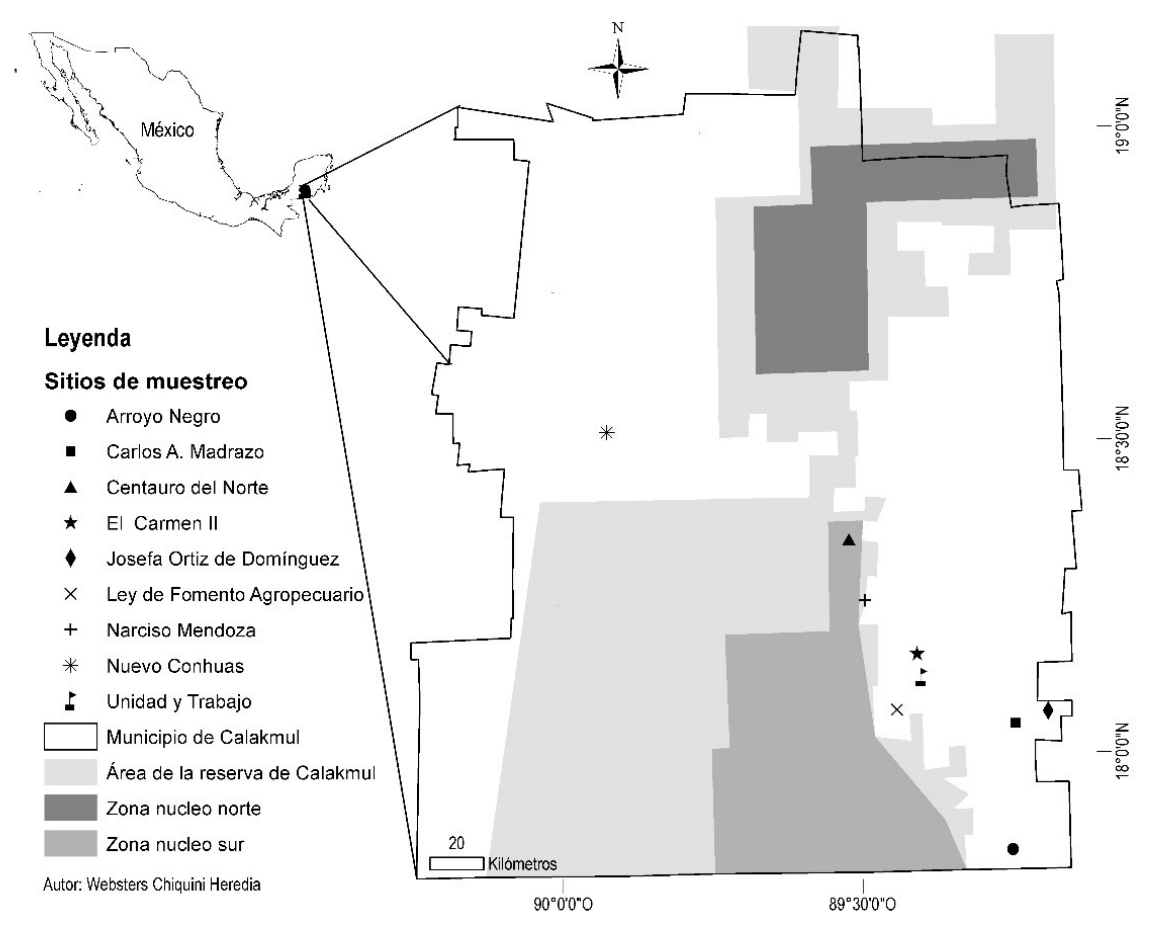

Figura 1. Ubicación geográfica de los sitios y su distribución en el municipio de Calakmul, en gris se muestra el área de amortiguamiento de la reserva y sus zonas núcleo.

$\mathrm{m}^{2}$ de $17.84 \mathrm{~m}$ de radio, con un círculo concéntrico de $400 \mathrm{~m}^{2}$ de $11.28 \mathrm{~m}$ de radio. En los círculos de $400 \mathrm{~m}^{2}$ se censaron todos los individuos que a 1.30 $\mathrm{m}$ del suelo tuvieran un diámetro normal (Dn) igual o mayor a $2.50 \mathrm{~cm}$ y a partir de los $10 \mathrm{~cm}$ en el resto del círculo de $1000 \mathrm{~m}^{2}$. Los individuos que cumplieron con los criterios se etiquetaron, registró la altura total $(\mathrm{m})$, el Dn $(\mathrm{cm})$, y su clasificación taxonómica de acuerdo con las claves respectivas.

\section{Análisis de la información}

Con la información de identidad taxonómica de campo, se generó un listado florístico con el orden, familia, género y especie. Se estimó la riqueza de especies, como el número de especies por unidad de muestreo. La diversidad se calculó con el índice de Shannon-Wiener $\left(\mathrm{H}^{\prime}\right)$, que permite conocer como varía la riqueza y la distribución de los individuos entre las especies. A partir de los valores de equitatividad (J') se comparó que tan bien se ajusta la diversidad observada con la diversidad máxima esperada, las cuales se calcularon con las fórmulas de Begon et al. (2006) y Magurran (2004):

$$
H^{\prime}=-\sum p i * \log P i
$$

Donde: $\mathrm{H}=$ Índice de Shannon-Wiener, $\mathrm{Pi}=$ Abundancia relativa, $\log =$ Logaritmo base 10 .

$$
\mathrm{J}^{\prime}=\frac{H^{\prime}}{H_{M A X}^{\prime}}
$$

Donde: $\mathrm{H}^{\prime}{ }_{M A X}=\ln \mathrm{S}, \mathrm{S}=$ Número de especies y $H^{\prime}=$ Índice de Shannon-Wiener.

Para determinar diferencias en la diversidad de los sitios se realizaron verificaciones por pares entre los nueve sitios con la prueba de t-student (Hutcheson 1970). Para estimar la similitud se empleó un análisis de agrupamiento con la medida de Bray-Curtis, para visualizar de forma gráfica la distribución de las parcelas de acuerdo a la similitud que comparten (Calderón-Mandujano et al. 2008). Los cálculos se realizaron con el programa Past ${ }^{\circledR}$ versión 2.17c (Hammer et al. 2001). También se 


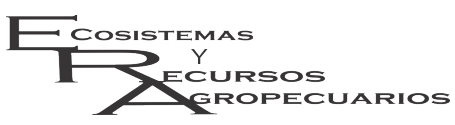

estimó la densidad como el número de individuos por unidad de área (individuos hectárea ${ }^{-1}$ ), el área basal $(\mathrm{Dn} / 2) 2 \pi$. El valor de importancia relativa (VIR) revela la importancia ecológica de cada especie en una comunidad vegetal, que utiliza parámetros estructurales básicos como abundancia relativa (AR) (individuos $\mathrm{ha}^{-1}$ ), frecuencia relativa(FR) y dominancia relativa (DR) $\left(\mathrm{m}^{2} \mathrm{ha}^{-1}\right)$ (Magurran 2004):

Donde:

$$
\mathrm{VIR}=\frac{A R+D R+F R}{3}
$$

$$
\begin{aligned}
& \mathrm{AR}=\frac{\text { No. de individuos por especie }}{\text { No. total de individuos de todas las especies }} \times 100 \\
& \mathrm{DR}=\frac{\text { Área basal de cada especie }}{\text { Área basal total de todas las especies }} \times 100 \\
& \mathrm{FR}=\frac{\text { Frecuencia de una especie }}{\text { Suma de la frecuencia de todas las especies }} \times 100
\end{aligned}
$$

El análisis de estructura horizontal (Dn) y vertical (altura) de la vegetación se realizó con las distribuciones de frecuencia agrupadas por clase, empleando la fórmula $\mathrm{K}=1+3.33 \times \log (n)$, donde $\mathrm{K}$ se refiere al número de clases y $\mathrm{n}$ el número de árboles. La diversidad estructural se estimó de la diversidad de especies, la altura y el área basal (Martínez-Sánchez 2016). Los indicadores evaluados fueron el índice de Shannon-Wiener para las especies (Hs), el índice de Shannon-Wiener para clases diamétricas (Hd), el índice de Shannon-Wiener para clases de altura $(\mathrm{Hh})$ y el índice promedio de la diversidad estructural (Hsdh), con las fórmulas:

$$
H s=\sum_{i=1}^{m} p i * \log p i
$$

Donde $\mathrm{pi}=$ proporción del área basal para la especie $\mathrm{i}, \mathrm{y} \mathrm{m}=$ número de especies

$$
H d=\sum_{i=1}^{d} p i * \log p i
$$

Donde pi= proporción del área basal por clase la diamétrica $\mathrm{i}, \mathrm{y} \mathrm{d}=$ número de clases diamétricas

$$
H h=\sum_{i=1}^{h} p i * \log p i
$$

Donde pi= proporción del área basal para la clase de altura $\mathrm{i}, \mathrm{y} \mathrm{h}=$ número de clases de altura

$$
H s d h=\frac{H s+H d+H h}{3}
$$

Donde $\mathrm{Hs}=$ diversidad por especies, $\mathrm{Hd}=$ diversidad por clases diamétricas, y $\mathrm{Hh}=$ diversidad por clases de altura. Para determinar diferencias significativas en la diversidad estructural de los sitios se realizaron verificaciones por pares entre los nueve sitios con la prueba de t-student (Hutcheson 1970).

\section{RESULTADOS}

\section{Composición de la comunidad}

Se midieron 2214 árboles, que pertenecen a 112 especies, 84 géneros, 36 familias y 21 órdenes en un área de 0.9 ha (Tabla 1 ). Las familias mejor representadas fueron Fabaceae (29 especies), Rubiaceae (10), Sapotaceae (7), Apocynaceae (6) y Polygonaceae (6). Estas cinco familias representan el $47 \%$ del total de especies registradas, el resto de las familias están constituidas por cinco o menos especies. De las 112 especies solamente tres $(2.68 \%)$ se comparten en los nueve sitios, las cuales fueron Hampea trilobata, M. brownei y Vitex gaumeri; y 32 especies $(28.57 \%)$ fueron especies únicas. De las especies únicas, NM contabilizó ocho especies $(12.50 \%)$ de la riqueza del sitio, mientras que AN $(25 \%)$ y EC (15\%) registraron seis especies, y LFA cinco especies $(8.93 \%)$. En el resto de los sitios se contabilizaron menos de tres especies, siendo UT el único sitio que no presentó especies únicas.

\section{Diversidad de especies y equitatividad}

Los sitios que tuvieron mayor riqueza fueron LFA y NM con 56 especies, seguido de $\mathrm{CN}$ con 51 especies $(45.53 \%)$, mientras que AN registró la menor riqueza con 24 especies $(21.43 \%)$. Los mayores valores de diversidad se obtuvieron en $\mathrm{CN}$ (1.558), LFA (1.537) y NM (1.503); mientras que AN (1.057) obtuvo el valor más bajo de diversidad. Los valores de equitatividad muestran que $\mathrm{CN}$ presentó la mejor distribución de abundancia entre las especies al ajustarse más de 0.9 la diversidad observada con la diversidad máxima esperada. 
Tabla 1. Listado de las especies arbóreas y sus abundancias por sitio de $1000 \mathrm{~m}^{2}$.

\begin{tabular}{|c|c|c|c|c|c|c|c|c|c|c|c|c|}
\hline Orden & Familia & Genero y Especie & G & 1 & 2 & 3 & 4 & 5 & 6 & 7 & 8 & 9 \\
\hline \multirow[t]{2}{*}{ Apiales } & Araliaceae & & & & & & & & & & & \\
\hline & & Dendropanaxarboreus & 1 & & & & & & & 1 & & \\
\hline \multirow[t]{4}{*}{ Arecales } & Arecaceae & & & & & & & & & & & \\
\hline & & Cryosophila stauracantha & & 5 & 49 & & 27 & 8 & & & & 18 \\
\hline & & Sabal mexicana & & & 3 & & 1 & 1 & & & & \\
\hline & & Sabal yapa & & & & & & & 1 & & & \\
\hline \multirow[t]{3}{*}{ Boraginales } & Boraginaceae & & & & & & & & & & & \\
\hline & & Bourreria pulchra & & & & & & & 1 & 1 & & 2 \\
\hline & & Cordia dodecandra & I & & & & & & & 1 & 1 & \\
\hline \multirow[t]{3}{*}{ Brassicales } & Capparaceae & & & & & & & & & & & \\
\hline & & Capparis indica & & & & 1 & & & 1 & 1 & & \\
\hline & & Quadrella cynophallophora & & & & & & & & 1 & & \\
\hline \multirow[t]{2}{*}{ Canellales } & Canellaceae & & & & & & & & & & & \\
\hline & & Canella winterana & & 1 & & & & & & & & \\
\hline \multirow[t]{10}{*}{ Caryophyllales } & Nyctaginaceae & & & & & & & & & & & \\
\hline & & Neea choriophylla & 1 & & & 2 & 1 & & 2 & & 1 & 2 \\
\hline & & Neea psychotrioides & 1 & & & & & & & 4 & & \\
\hline & Polygonaceae & & & & & & & & & & & \\
\hline & & Coccoloba acapulcensis & 1 & & 1 & 1 & 9 & & 2 & 2 & 3 & \\
\hline & & Coccoloba barbadensis & 1 & & & & & & 2 & 3 & 1 & 1 \\
\hline & & Coccoloba cozumelensis & 1 & & & 7 & & 13 & 2 & 11 & 2 & \\
\hline & & Coccoloba reflexiflora & 1 & & 6 & 16 & & & 20 & 14 & 29 & 13 \\
\hline & & Gymnopodium floribundum & $\mathrm{S}$ & & & 7 & 3 & & 6 & & 7 & 3 \\
\hline & & Neomillspaughia emarginata & & & & & & & 1 & & & \\
\hline \multirow[t]{4}{*}{ Celastrales } & Celastraceae & & & & & & & & & & & \\
\hline & & Maytenus schippii & $\mathrm{S}$ & & 1 & 1 & 4 & & & & & 1 \\
\hline & & Rhacoma gaumeri & & & & 3 & & & & & & 2 \\
\hline & & Semialarium mexicanum & I & 1 & 3 & 3 & & & 2 & 2 & 2 & 1 \\
\hline \multirow[t]{14}{*}{ Ericales } & Ebenaceae & & & & & & & & & & & \\
\hline & & Diospyros bumelioides & $\mathrm{S}$ & & & & & & & 1 & & \\
\hline & & Diospyros salicifolia & $\mathrm{S}$ & & & 6 & & & 4 & 1 & 1 & 7 \\
\hline & Primulaceae & & & & & & & & & & & \\
\hline & & Bonellia flammea & & & & & & & 2 & & & 1 \\
\hline & & Bonellia macrocarpa & & & 2 & 2 & 1 & & & 3 & & \\
\hline & & Parathesis cubana & & & & 1 & & & 6 & 6 & & \\
\hline & Sapotaceae & & & & & & & & & & & \\
\hline & & Manilkara zapota & $\mathrm{S}$ & & 11 & 12 & 24 & 19 & 6 & 11 & 13 & 24 \\
\hline & & Pouteria campechiana & $\mathrm{S}$ & & 1 & & & 5 & & & & \\
\hline & & Pouteria reticulata & $\mathrm{S}$ & & 8 & 8 & 40 & 2 & 3 & & & 21 \\
\hline & & Sideroxylon obtusifolium & 1 & & & & & & & & 1 & \\
\hline & & Sideroxylon rotundifolium & 1 & & & & & & 1 & & & \\
\hline & & Sideroxylon salicifolium & I & & & & & & & 1 & & \\
\hline \multirow[t]{17}{*}{ Fabales } & Fabaceae & & & & & & & & & & & \\
\hline & & Acacia centralis & $\mathrm{P}$ & & & & & & 1 & 3 & & \\
\hline & & Acacia cornigera & $\mathrm{P}$ & 2 & 3 & & 1 & 5 & 1 & & & \\
\hline & & Acacia gaumeri & $\mathrm{P}$ & & & & & & & 2 & & \\
\hline & & Acacia angustissima & $\mathrm{P}$ & & & 1 & & & & 1 & & \\
\hline & & Acacia globulifera & $\mathrm{P}$ & 1 & 1 & & & & & & & \\
\hline & & Ateleia gummifera & $\mathrm{P}$ & & & 5 & & & 7 & 2 & 3 & \\
\hline & & Bauhinia divaricata & & 1 & & & & & & & & \\
\hline & & Caesalpinia gaumeri & I & & & & & & & & 2 & \\
\hline & & Caesalpinia mollis & 1 & & & & 3 & & & 1 & & 1 \\
\hline & & Caesalpinia yucatanensis & I & & & & & & & & 2 & \\
\hline & & Calliandra belizensis & & & & & & & 24 & & & \\
\hline & & Chloroleucon mangense & & 14 & & & & & & & & \\
\hline & & Croton arboreus & I & & & & 7 & & & & & \\
\hline & & Croton icche & I & 2 & 26 & 14 & & 4 & 24 & 28 & 12 & 16 \\
\hline & & Diphysa carthagenensis & $\mathrm{P}$ & & & 1 & & & & 1 & & \\
\hline & & Gliricidia maculata & $\mathrm{P}$ & & & 2 & & & 10 & 1 & 3 & 6 \\
\hline
\end{tabular}


Chiquini-Heredia et al.

Tabla 1. Continuación

\begin{tabular}{|c|c|c|c|c|c|c|c|c|c|c|c|c|}
\hline Orden & Familia & Genero y Especie & G & 1 & 2 & 3 & 4 & 5 & 6 & 7 & 8 & 9 \\
\hline & & Gymnanthes lucida & 1 & & 35 & & & & & 25 & & \\
\hline & & Haematoxylum campechianum & I & & 2 & 6 & 2 & & 7 & 10 & 17 & 3 \\
\hline & & Havardia albicans & & & & 2 & & & 3 & 4 & 3 & 2 \\
\hline & & Jatropha gaumeri & & & & 5 & & 6 & 5 & 16 & 4 & 6 \\
\hline & & Lonchocarpus castilloi & 1 & & 5 & & 3 & 1 & & & & \\
\hline & & Lonchocarpus guatemalensis & 1 & 5 & 6 & 12 & 2 & 1 & 7 & 2 & 18 & \\
\hline & & Lonchocarpus hondurensis & 1 & 11 & & & & & & & & \\
\hline & & Lonchocarpus rugosus & 1 & & & 1 & & & & & & \\
\hline & & Lysiloma latisiliquum & I & & & & & & & 1 & & \\
\hline & & Mimosa bahamensis & $\mathrm{P}$ & & & 3 & & & 6 & & 2 & 1 \\
\hline & & Platymiscium yucatanum & 1 & & & & 1 & & & & 1 & \\
\hline & & Sebastiania adenophora & & 44 & & 6 & & & 3 & & & 6 \\
\hline & & Swartzia cubensis & 1 & & 2 & & & 2 & 1 & 1 & & 3 \\
\hline \multirow{14}{*}{ Gentianales } & Apocynaceae & & & & & & & & & & & \\
\hline & & Aspidosperma desmanthum & I & & & & & 1 & & & & 4 \\
\hline & & Cameraria latifolia & & & 5 & & 4 & 2 & & 8 & 23 & \\
\hline & & Cascabela gaumeri & $\mathrm{P}$ & & & 1 & 4 & & & & & \\
\hline & & Plumeria obtusa & & & & & & & 5 & 1 & 4 & 1 \\
\hline & & Thevetia ahouai & $\mathrm{P}$ & & 1 & & & & & & & \\
\hline & Rubiaceae & & & & & & & & & & & \\
\hline & & Alseis yucatanensis & $\mathrm{S}$ & & & & 1 & & & & & \\
\hline & & Guettarda combsii & 1 & & & & & & 1 & & & \\
\hline & & Guettarda gaumeri & I & & & 9 & & 1 & 9 & 3 & 10 & \\
\hline & & Machaonia lindeniana & & & & 1 & & & 1 & 9 & 7 & \\
\hline & & Randia aculeata & I & & & 11 & & & 2 & 4 & 5 & \\
\hline & & Randia longiloba & I & 25 & & 2 & 1 & 1 & 2 & & & 6 \\
\hline & & Simira salvadorensis & 1 & 1 & 1 & & 2 & 2 & 1 & & & \\
\hline \multirow[t]{7}{*}{ Lamiales } & Acanthaceae & & & & & & & & & & & \\
\hline & & Bravaisia berlandieriana & I & & & & & & & 1 & & \\
\hline & Bignoniaceae & & & & & & & & & & & \\
\hline & & Handroanthus chrysanthus & I & & & & 2 & & & & & \\
\hline & & Tabebuia rosea & I & 10 & & & & & & & & \\
\hline & Lamiaceae & & & & & & & & & & & \\
\hline & & Vitex gaumeri & I & 1 & 2 & 1 & 2 & 1 & 3 & 1 & 5 & 3 \\
\hline \multirow[t]{5}{*}{ Laurales } & Lauraceae & & & & & & & & & & & \\
\hline & & Licaria campechiana & & & & & & & 6 & & & 1 \\
\hline & & Licaria coriacea & & & & & & 1 & & & & \\
\hline & & Licaria peckii & & & & & & 4 & & & & 1 \\
\hline & & Nectandra salicifolia & I & & & 14 & 21 & & & 4 & & 2 \\
\hline Magnoliales & Annonaceae & & & & & & & & & & & \\
\hline & & Mosannona depressa & I & & 2 & 3 & 2 & & 1 & 2 & 1 & 3 \\
\hline \multirow{11}{*}{ Malpighiales } & Clusiaceae & & & & & & & & & & & \\
\hline & & Calophyllum brasiliense & I & & 7 & & & 7 & & & & 2 \\
\hline & Erythroxylaceae & & & & & & & & & & & \\
\hline & Malpighiaceae & Erythroxylum rotundifolium & 1 & & & 5 & & & 9 & & 21 & \\
\hline & & Byrsonima bucidifolia & & & 1 & 6 & 2 & & 1 & 4 & 1 & \\
\hline & & Malpighia glabra & & & 1 & 4 & & & 4 & 3 & 3 & \\
\hline & Putranjivaceae & & & & & & & & & & & \\
\hline & & Drypetes lateriflora & I & & & 11 & 33 & & & & & \\
\hline & Salicaceae & & & & & & & & & & & \\
\hline & & Laetia thamnia & I & & 1 & & & 1 & 4 & 5 & & \\
\hline & & Zuelania guidonia & $\mathrm{S}$ & 1 & & & & 2 & 1 & & & \\
\hline \multirow[t]{3}{*}{ Malvales } & Malvaceae & & & & & & & & & & & \\
\hline & & Hampea trilobata & 1 & 4 & 1 & 2 & 5 & 1 & 5 & 14 & 1 & 2 \\
\hline & & Pseudobombax ellipticum & 1 & & 2 & & & 1 & & & & 1 \\
\hline \multirow{2}{*}{ Myrtales } & Con & & & & & & & & & & & \\
\hline & & Bucida buceras & I & 2 & 8 & 3 & & 4 & & 1 & 1 & \\
\hline
\end{tabular}


Tabla 1. Continuación.

\begin{tabular}{|c|c|c|c|c|c|c|c|c|c|c|c|c|}
\hline Orden & Familia & Genero y Especie & G & 1 & 2 & 3 & 4 & 5 & 6 & 7 & 8 & 9 \\
\hline \multirow[t]{2}{*}{ Ranunculales } & Menispermaceae & & & & & & & & & & & \\
\hline & & Hyperbaena winzerlingii & & & & 4 & 3 & & 11 & 8 & 7 & 11 \\
\hline \multirow[t]{5}{*}{ Rosales } & Moraceae & & & & & & & & & & & \\
\hline & & Ficus cotinifolia & I & 1 & & & & & & 1 & & \\
\hline & & Trophis racemosa & I & 7 & & & & 1 & & & & \\
\hline & Rhamnaceae & & & & & & & & & & & \\
\hline & & Krugiodendron ferreum & I & 1 & 4 & 14 & 9 & 1 & & & 1 & 2 \\
\hline \multirow[t]{6}{*}{ Rosanae } & Myrtaceae & & & & & & & & & & & \\
\hline & & Eugenia capuli & I & & & & & & 5 & & & 1 \\
\hline & & Eugenia ibarrae & I & & 7 & 24 & 17 & 2 & 26 & 12 & 43 & 6 \\
\hline & & Eugenia winzerlingii & I & & & 4 & & & 22 & 26 & & \\
\hline & & Myrciaria floribunda & I & & 3 & 8 & 25 & & & & & 12 \\
\hline & & Pimenta dioica & I & & & & 1 & & & & & \\
\hline \multirow[t]{3}{*}{ Santalales } & Opiliaceae & & & & & & & & & & & \\
\hline & & Agonandra obtusifolia & & & & 3 & & & & & & \\
\hline & & Agonandra ovatifolia & & & & & 3 & & & & & \\
\hline \multirow[t]{15}{*}{ Sapindales } & Anacardiaceae & & & & & & & & & & & \\
\hline & & Metopium brownei & 1 & 2 & 9 & 19 & 2 & 5 & 7 & 17 & 9 & 17 \\
\hline & Burseraceae & & & & & & & & & & & \\
\hline & & Bursera simaruba & I & & & 5 & 3 & 1 & 16 & 3 & 2 & 4 \\
\hline & & Protium copal & I & & 1 & & 2 & & & & & 1 \\
\hline & Meliaceae & & & & & & & & & & & \\
\hline & Rutaceae & Swietenia macrophylla & I & & 1 & & 1 & 1 & 2 & 2 & & 3 \\
\hline & & Amyris elemifera & I & & & 9 & 2 & & 5 & 7 & 1 & 4 \\
\hline & Sapindaceae & & & & & & & & & & & \\
\hline & & Cupania belizensis & I & 3 & & & & & & & & \\
\hline & & Exothea diphylla & & & & 4 & & & 2 & & & \\
\hline & & Matayba oppositifolia & & 4 & & & & 36 & 1 & 1 & & 12 \\
\hline & & Melicoccus oliviformis & & & & & 1 & & & & & \\
\hline & & Talisia floresii & I & & & 3 & & & & 1 & & \\
\hline & Total de individu & por sitio & & 149 & 222 & 298 & 277 & 143 & 313 & 300 & 273 & 239 \\
\hline
\end{tabular}

Mientras que AN y CAM son los sitios que tuvieron los menores valores de equitatividad, lo que indica mayor abundancia de alguna especie, por los valores menores a 0.8 (Tabla 2). Los sitios muestran diferencias significativas $(p>0.05)$ en sus valores de diversidad, con excepción de CAM con EC ( $\mathrm{t}=$ 1.51) y JOD ( $\mathrm{t}=0.44) ; \mathrm{CN}$ con LFA $(\mathrm{t}=0.62)$ y $\mathrm{NM}(\mathrm{t}=1.80)$; $\mathrm{EC}$ con JOD $(\mathrm{t}=1.78)$ y $\mathrm{NC}(\mathrm{t}=$ $0.92)$; así como LFA y NM ( $\mathrm{t}=1.12)$, NM con UT $(\mathrm{t}=1.81)$.

\section{Similitud en composición de especies}

El análisis de agrupamiento $(r=0.8812)$ mostró que la SI localizada en AN es diferente de las otras $\mathrm{SI}$, lo que indica que la composición de especies es diferente (Figura 2). Además, se observa que las SI con mayor similitud (mayor al
$50 \%$ son las que se localizan en CN, LFA, NC y NM. El sitio CN comparte 37 especies, $72.55 \%$ de la riqueza del sitio con el sitio LFA (66.07\%), 32 especies con NC (80\%) y 35 especies con NM $(62.5 \%)$. En tanto que el sitio LFA comparte 32 especies, $57.14 \%$ de las especies con NC (80 \%) y 37 especies con NM (66.07\%). Mientras que el sitio NC comparte 31 especies, $77.5 \%$ de la riqueza en el sitio con NM (55.36\%). Por otro lado, las SI de CAM, EC y UT forman un grupo, a un nivel de similitud menor al $40 \%$.

\section{Estructura de la vegetación}

La mayor densidad de individuos se presentó en las SI de LFA, seguida de NM y CN, mientras los valores más bajos se presentaron en JOD y AN. Los valores más altos de área basal se presentaron en las 
Tabla 2. Parámetros estructurales de los sitios.

\begin{tabular}{|c|c|c|c|c|c|}
\hline Sitio & $\mathrm{D}\left(\right.$ Ind. ha $\left.{ }^{-1}\right)$ & $A B\left(m^{2} h a^{-1}\right)$ & $\mathrm{S}$ & $\mathrm{H}^{\prime} \log 10$ & $J^{\prime}$ \\
\hline Ley de Fomento Agropecuario & 7285 & 11.071 & 56 & 1.537 & 0.879 \\
\hline Narciso Mendoza & 7065 & 10.283 & 56 & 1.503 & 0.86 \\
\hline Centauro del Norte & 6855 & 13.225 & 51 & 1.558 & 0.912 \\
\hline Unidad y Trabajo & 5155 & 15.127 & 44 & 1.435 & 0.873 \\
\hline El Carmen II & 6500 & 13.741 & 40 & 1.294 & 0.808 \\
\hline Nuevo Conhuás & 5985 & 14.989 & 40 & 1.331 & 0.831 \\
\hline Carlos A. Madrazo & 4790 & 18.224 & 36 & 1.229 & 0.79 \\
\hline Josefa Ortiz de Domínguez & 2810 & 16.128 & 33 & 1.219 & 0.803 \\
\hline Arroyo Negro & 3455 & 5.629 & 24 & 1.057 & 0.766 \\
\hline
\end{tabular}

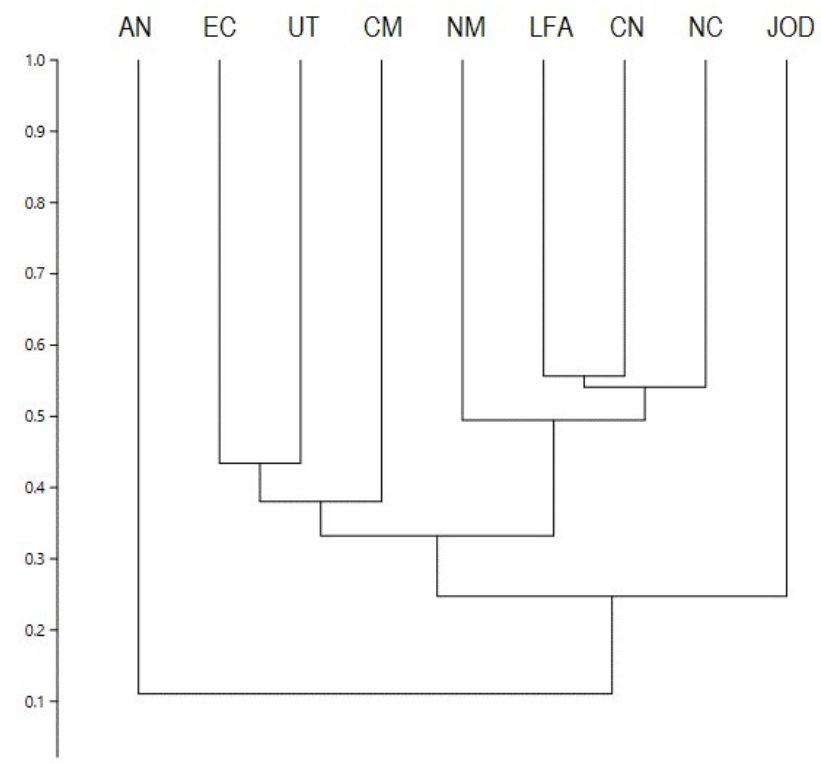

Figura 2. Análisis de agrupamiento calculado con el índice de Bray-Curtis.

SI de CAM, JOD, UT y NC, mientras que en AN se estimaron las menores áreas basales. Por otro lado, las especies con mayores VIR fueron S. adenophora en AN, M. zapota en CN y EC, M. oppositifolia en JOD, $C$. reflexiflora en LFA, $M$. brownei en UT, G. lucida en $\mathrm{CM}$ y $\mathrm{H}$. campechianum en NC y NM (Tabla 3).

El análisis de la estructura horizontal mostró que, en todas las unidades de muestreo, la mayor proporción de individuos (más del 70 \%) se agrupó dentro de las tres primeras clases diamétricas $\leq 3$, $>3$ a 6 y 6 a $9 \mathrm{~cm}$, en todos los sitios. Las curvas presentan la forma característica de J-invertida, es decir que hay una baja proporción de individuos con Dn grandes y una alta proporción de individuos pequeños, lo que indica el potencial de regeneración de la vegetación a partir del reclutamiento de individuos (Figura 3).

La altura de los individuos estuvo en el rango de 3 a $13 \mathrm{~m}$, con más del $80 \%$ de los individuos en la tercera y sexta categoría, con distribución en forma de campana (Figura 4).

\section{Diversidad estructural}

Al comparar los índices de diversidad estructural $\mathrm{Hs}, \mathrm{Hd}$, Hh y Hsdh, se encontró que AN 
Tabla 3. Lista de las especies que alcanzaron mayor VIR en cada sitio, el corte se realizó al alcanzar el $30 \%$.

\begin{tabular}{|c|c|c|c|c|c|}
\hline Sitio & Taxón & Ab. Rel. & Dom. Rel. & Frec. Rel. & VIR \\
\hline \multirow[t]{2}{*}{ Arroyo Negro } & Sebastiania adenophora & 29.53 & 37.54 & 1.05 & 22.71 \\
\hline & $\begin{array}{l}\text { Lonchocarpus hondurensis } \\
\text { Otras } 22 \text { especies }\end{array}$ & 7.38 & 19.16 & 0.26 & 8.93 \\
\hline \multirow[t]{4}{*}{ Carlos A. Madrazo } & Gymnanthes lucida & 15.77 & 30.87 & 0.53 & 15.72 \\
\hline & Cryosophila stauracantha & 22.07 & 4.12 & 1.32 & 9.17 \\
\hline & Croton icche & 11.71 & 4.12 & 2.11 & 5.98 \\
\hline & Otras 33 especies & & & & \\
\hline \multirow[t]{5}{*}{ Centauro del Norte } & Manilkara zapota & 4.03 & 16.59 & 2.11 & 7.58 \\
\hline & Metopium brownei & 6.38 & 11.44 & 2.37 & 6.73 \\
\hline & Haematoxylum campechianum & 2.01 & 15.61 & 1.84 & 6.49 \\
\hline & Coccoloba reflexiflora & 5.37 & 7.33 & 1.58 & 4.76 \\
\hline & Eugenia ibarrae & 8.05 & 3.00 & 2.11 & 4.39 \\
\hline \multirow{4}{*}{ El Carmen II } & Manilkara zapota & 8.66 & 26.71 & 2.11 & 12.49 \\
\hline & Drypetes lateriflora & 11.91 & 13.89 & 0.53 & 8.78 \\
\hline & Pouteria reticulata & 14.44 & 7.24 & 1.58 & 7.75 \\
\hline & $\begin{array}{l}\text { Cryosophila stauracantha } \\
\text { Otras } 36 \text { especies }\end{array}$ & 9.75 & 3.19 & 1.32 & 4.75 \\
\hline \multirow[t]{4}{*}{ Josefa Ortiz de Domínguez } & Matayba oppositifolia & 25.17 & 25.04 & 1.32 & 17.18 \\
\hline & Bucida buceras & 2.80 & 25.27 & 1.58 & 9.88 \\
\hline & Coccoloba cozumelensis & 9.09 & 5.46 & 1.32 & 5.29 \\
\hline & Otras 30 especies & & & & \\
\hline \multirow[t]{7}{*}{ Ley de Fomento Agropecuario } & Coccoloba reflexiflora & 6.39 & 14.96 & 1.58 & 7.64 \\
\hline & Metopium brownei & 2.24 & 11.68 & 2.37 & 5.43 \\
\hline & Eugenia winzerlingii & 7.03 & 2.06 & 0.79 & 3.29 \\
\hline & Calliandra belizensis & 7.67 & 3.24 & 0.26 & 3.72 \\
\hline & Eugenia ibarrae & 8.31 & 3.13 & 2.11 & 4.52 \\
\hline & Bursera simaruba & 5.11 & 6.42 & 1.84 & 4.46 \\
\hline & Hyperbaena winzerlingii & 3.51 & 7.71 & 1.58 & 4.27 \\
\hline \multirow[t]{6}{*}{ Narciso Mendoza } & Haematoxylum campechianum & 3.33 & 17.95 & 1.84 & 7.71 \\
\hline & Metopium brownei & 5.67 & 13.92 & 2.37 & 7.32 \\
\hline & Gymnanthes lucida & 8.33 & 3.21 & 0.53 & 4.02 \\
\hline & Eugenia winzerlingii & 8.67 & 1.53 & 0.79 & 3.66 \\
\hline & Croton icche & 9.33 & 5.62 & 2.11 & 5.69 \\
\hline & Otras 51 especies & & & & \\
\hline \multirow[t]{5}{*}{ Nuevo Conhuás } & Haematoxylum campechianum & 6.23 & 25.22 & 1.84 & 11.10 \\
\hline & Coccoloba reflexiflora & 10.62 & 14.89 & 1.58 & 9.03 \\
\hline & Cameraria latifolia & 8.42 & 8.48 & 1.32 & 6.07 \\
\hline & Erythroxylum rotundifolium & 7.69 & 9.78 & 0.79 & 6.09 \\
\hline & Otras 36 especies & & & & \\
\hline \multirow[t]{4}{*}{ Unidad y Trabajo } & Metopium brownei & 7.11 & 23.52 & 2.37 & 11 \\
\hline & Manilkara zapota & 10.04 & 15.22 & 2.11 & 9.12 \\
\hline & Hyperbaena winzerlingii & 4.60 & 9.73 & 1.58 & 5.30 \\
\hline & Coccoloba reflexiflora & 5.44 & 8.66 & 1.58 & 5.23 \\
\hline
\end{tabular}

presenta en todos los casos los valores de diversidad estructural bajos (Tabla 4). En el caso de $\mathrm{Hs}$, AN presenta valores significativamente menores $(p>0.001)$ que el resto de los sitios analizados, mientras que NM y LFA presentan un valor significativamente mayor $(p>0.001)$ que UT. Para los valores de $\mathrm{Hd}$, no hay diferencias significativas entre las SI. Los valores de $\mathrm{Hh}$ muestran que JOD tiene un valor significativamente mayor $(p>0.001)$ que
CM, AN y CN. En el caso de la Hsdh los valores más altos se presentaron en NM y LFA, teniendo los valores más bajos en AN y CM.

\section{DISCUSIÓN}

\section{Composición de la comunidad}

Las familias con mayor número de especies coinciden con los reportes para selvas subperennifo- 


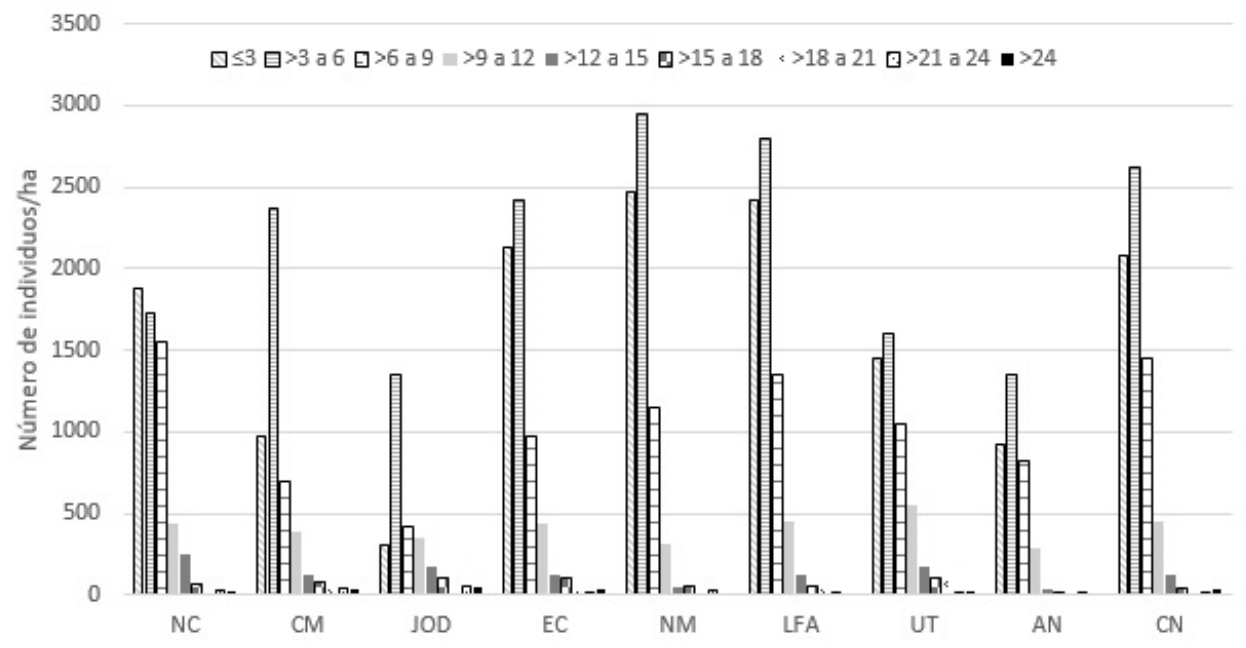

Figura 3. Distribución de los individuos por clases diamétricas en cada uno de los sitios.

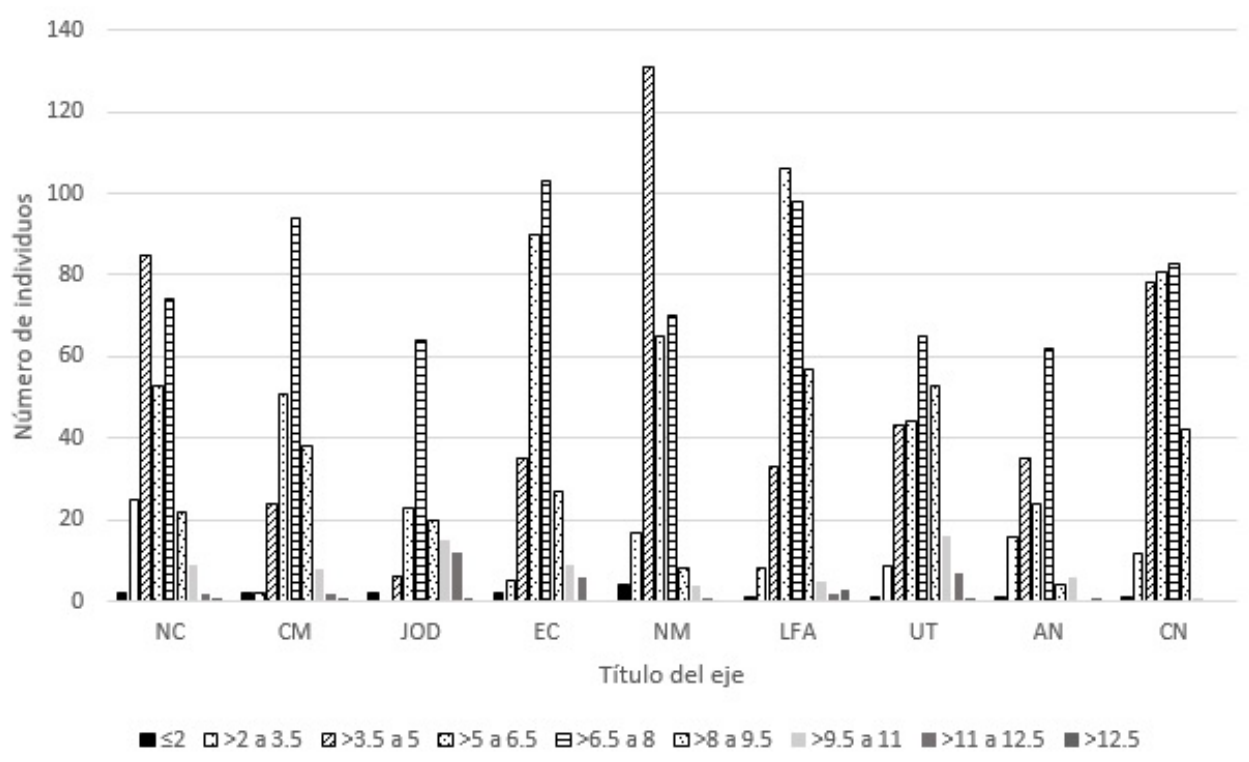

Figura 4. Distribución vertical de los individuos en cada uno de los sitios.

lias de la península de Yucatán (Zamora-Crescencio et al. 2008, Zamora-Crescencio et al. 2012, GarcíaLicona et al. 2014) y otros lugares (DamascenoJunior et al. 2005). El número de especies es mayor que el reportado para otras selvas inundables de México (Cortés-Castelán e Islebe 2005, ZamoraCrescencio et al. 2008, Maldonado-Sánchez et al. 2016). La familia Fabaceae es importante por su alto número de especies (Martínez y Galindo-Leal
2002), en este trabajo se registró mayor número de especies de las que obtuvo Zamora-Crescencio et al. (2008) y Maldonado-Sánchez et al. (2016) en selvas del sur de México, pero el número de especies es menor que las reportadas por GonzálezValdivia et al. (2012). Las diferencias antes mencionadas pueden deberse a que las especies de la familia Fabaceae son indicadoras de vegetación se- 
Tabla 4. Valores de diversidad estructura por sitio.

\begin{tabular}{lcccc}
\hline Sitio & $\mathrm{Hs}$ & $\mathrm{Hd}$ & $\mathrm{Hh}$ & $\mathrm{Hsdh}$ \\
\hline Ley de Fomento Agropecuario & 1.400 & 0.882 & 0.716 & 0.999 \\
Narciso Mendoza & 1.379 & 0.909 & 0.722 & 1.003 \\
Centauro del Norte & 1.310 & 0.883 & $0.591^{b}$ & 0.928 \\
Unidad y Trabajo & 1.212 & 0.877 & 0.714 & 0.934 \\
El Carmen II & 1.169 & 0.899 & 0.730 & 0.932 \\
Nuevo Conhuás & 1.143 & 0.886 & 0.730 & 0.919 \\
Carlos A. Madrazo & 1.097 & 0.844 & $0.646^{b}$ & 0.862 \\
Josefa Ortiz de Domínguez & 1.053 & 0.846 & $0.803^{a}$ & 0.901 \\
Arroyo Negro & $0.908 \mathrm{a}$ & 0.818 & $0.630^{b}$ & 0.785 \\
\hline
\end{tabular}

cundaria y las selvas inundables estudiadas es muy probable que hayan estado expuestas a la extracción de madera y chicle (Martínez y Galindo Leal 2002, Kepleis 2004). Las 112 especies que se identificaron, tienen su distribución natural dentro de los límites de la república mexicana (CONABIO 2015), de las cuales C. stauracantha y Handroanthus chrysanthus se encuentran dentro de la categoría de riesgo, bajo el estatus Amenazada en la Norma Oficial Mexicana 059 (SEMARNAT 2010). Las especies Ateleia gummifera y $V$. gaumeri se encuentran en la categoría de riesgo bajo en el estatus de en peligro de extinción de la Unión Internacional para la Conservación de la Naturaleza (IUCN 2015). Mientras que la especie Swietenia macrophylla se encuentra en la Categoría II de la Convención sobre el Comercio Internacional de Especies Amenazadas de Fauna y Flora Silvestres (CITES 2015). Estas especies son de interés nacional e internacional por su status de conservación o control de su explotación, por lo que pueden tomarse como base para generar estrategias de manejo y conservación en las áreas de selva inundable. De las 112 especies se determinó el gremio al que pertenecen 80 especies, de los cuales 11 son pioneras (9.82\%), 60 intermedias ( $53.57 \%$ ) y 9 tolerantes a la sombra (8.04\%), en los nueve sitios estudiados hay presencia de los tres tipos de especies (Tabla 1). Las especies Haematoxylum campechianum, Cameraria latifolia, Tabebuia rosea, Erythroxylum rotundifolium, Myrciaria floribunda y Ficus cotinifolia, presentes en las SI estudiadas, se han reportado como especies características de selvas inundables por Martínez y Galindo Leal (2002), Vázquez Negrín et al. (2010), Maldonado-Sánchez et al. (2016).

\section{Diversidad de especies y equitatividad}

La mayor riqueza registrada por sitio, fue de 56 especies en LFA y NM, que es menor a las 65 especies reportadas por Díaz-Gallegos et al. (2002), para el ejido La Guadalupe en un área de 0.1 ha, pero supera a las 22 especies reportadas por Vázquez-Negrín et al. (2010); lo que puede deberse a la diferencia en los criterios utilizados en la medición de los individuos. El sitio de muestreo del ejido NC registró una riqueza de 40 especies en una superficie de 0.1 ha, similar a la riqueza reportada por Tun-Dzul et al. (2008) de 39 especies en una superficie de 0.05 ha en la misma comunidad. Aunque la cantidad de especies que se comparten es superior al $50 \%$ para la mayoría de los sitios, la abundancia de las especies influye en los resultados de las pruebas estadísticas al comparar la diversidad. La menor diversidad que presentan los sitios al sur del municipio podría deberse a la mayor precipitación (Martínez y Galindo-Leal 2002) que junto con las características topográficas del sitio y de las áreas que la circundan influyen en la inundación (Palacio-Aponte et al. 2002, Cortés-Castelán e Islebe 2005). En los sitios AN, JOD y CM al sur del municipio, se presentaron especies con una alta dominancia, como S. adenophora, Matayba oppositifolia y Chryosophila stauracantha respectivamente. La dominancia de estas especies podría estar relacionado con lo que indican Martínez y Galindo Leal (2002) sobre la influencia que tienen las diferencias en los rangos de humedad al sur del municipio, lo que permite a las especies con mejor capacidad de adaptación crear comunidades dominantes. 


\section{Similitud en composición de especies}

Las especies H. campechianum, Cameraria latifolia, Bucida buceras y M. brownei se encuentran distribuidas entre los sitios y son indicadoras de terrenos inundados en la región de Calakmul (Palacio-Aponte et al. 2002). Las mayores similitudes se obtuvieron en los sitios que se ubican al centro del municipio. Al respecto Martínez y Galindo-Leal (2002) indican que en el centro del municipio de Calakmul, se tienen condiciones homogéneas de humedad y topografía, lo que permite que se tengan condiciones para el establecimiento de un mayor número de especies. En los sitios al sur se tienen las menores similitudes sobresaliendo los sitios AN y JOD, con una composición de especies diferente, en los cuales no está presente $H$. campechianum, lo que indica que probablemente estos sitios tienen mayores profundidades de inundación o corrientes superficiales. En AN no se registraron especies de la familia Sapotaceae, probablemente por la composición del suelo (Cortés Castelán e Islebe 2005) o por la poca disponibilidad de oxigeno (López 2009). Al respecto Martínez y Galindo-Leal (2002) mencionan que las diferencias en la abundancia de especies tienen importancia ecológica al permitir que una amplia variedad de fauna pueda hacer uso de estas extensiones de SI, y coincide con Palacio-Aponte et al. (2002) quienes plantean el potencial que tiene la SI de Calakmul como humedal terrestre.

\section{Estructura de la vegetación}

Los valores de las áreas basales en los sitios son menores a lo reportados por MaldonadoSánchez et al. (2016) para selvas inundables de Tabasco, cercanos a lo que reportan Cortés Castelán e Islebe (2005) para selvas inundables de Quintana Roo y Zamora-Crescencio et al. (2008) para Calakmul. Las especies $H$. campechianum, M. zapota y $M$. brownei tienen los primeros lugares de VIR por los valores de dominancia que tuvieron, lo que indica que son individuos grandes. Estas especies también fueron reportadas como importantes por Díaz-Gallegos et al. (2002) y Palacio-Aponte et al. (2002). Las especies G. lucida, M. oppositifolia y
S. adenophora, no se reportan en otros trabajos, a pesar de que ocupan los primeros lugares de VIR en este estudio para los sitios del sur de Calakmul. La vegetación no presentó diferencias en los patrones de la estructura horizontal y vertical entre los sitios del centro y sur, los individuos más grandes están representados en las clases pequeñas, lo que indica una buena condición de los sitios estudiados.

\section{Diversidad estructural}

En general, los sitios NM, JOD y LFA fueron los más diversos estructuralmente, mientras que el sitio AN fue el de menor diversidad estructural. La diversidad estructural asociada con las clases diamétricas ( $\mathrm{Hd})$ no tuvo diferencias significativas entre los sitios, al contrario de las clases de alturas $(\mathrm{Hh})$, lo que puede estar asociado a que el atributo de altura puede estar influenciado por la inundación del sitio, pues se sabe que modifica las propiedades del suelo reduciendo la disponibilidad de oxígeno, lo que impacta en el crecimiento de los individuos y la capacidad del suelo para soportar individuos grandes (López 2009).

\section{CONCLUSIONES}

El tipo de vegetación en los sitios es de selva baja subperennifolia inundable. Las especies indicadoras de este tipo de vegetación como $H$. campechianum y $C$. latifolia, entre otras fueron reportadas para este trabajo, detectando especies con altos valores de importancia que no han sido reportados para la selva de Calakmul, como G. lucida, M. oppositifolia y $S$. adenophora. La diversidad estructural indica que la altura está influenciada por las inundaciones. La selva inundable de Calakmul tiene potencial para desarrollar programas de conservación, debido a que cuenta con especies prioritarias de atención en los programas nacionales e internacionales y a la importancia que tiene como aporte de agua y alimento a la fauna de la región.

\section{AGRADECIMIENTOS}

A los parataxónomos Demetrio Álvarez y 
Manuel Arana, por el apoyo en la identificación del material botánico en campo. Al Consejo Nacional de Ciencia y Tecnología por la beca de posgrado Número 354871 otorgada al primer autor. A los comisarios ejidales de las localidades en las cuales se trabajó. A Investigación y Soluciones Socioambientales A.C. por el apoyo en la logística y trabajo de campo.

\section{LITERATURA CITADA}

Begon M, Townsend CR, Harper JL (2006) Ecology from individuals to ecosystems. 4th edition. Malden, MA: Blackwell Publishing Ltd. 759p.

Calderón-Mandujano RR, Galindo-Leal C, Cedeño-Vazquez JR (2008) Utilización de hábitat por reptiles en estados sucesionales de selvas tropicales de Campeche, México. Acta Zoológica Mexicana 24: 95-114.

CITES (2015) Convention on International Trade in Endangered Species of Wild Fauna and Flora. Appendices I, II and III. UNEP, International Environment House, Switzerland. 46p.

CONABIO (2015) NaturaLista. Comisión Nacional para el Conocimiento y Uso de la Biodiversidad. http://na turalista.conabio.gob.mx/places/mexico\#establishment_means=endemic. Fecha de consulta 5 de mayo de 2015.

Cortés-Castelán JC, Islebe GA (2005) Influencia de factores ambientales en la distribución de especies arbóreas en las selvas del sureste de México. Revista de Biología tropical 53: 115.133.

Damasceno-Junior GA, Semir J, Dos Santos FAM, de Freitas Leitão-Filho H (2005) Structure, distribution of species y inundation in a riparian forest of Rio Paraguai, Pantanal, Brazil. Flora 200: 119-135.

Díaz-Gallegos JR, Castillo-Acosta O, García-Gil G (2002) Distribución espacial y estructura arbórea de la selva baja subperennifolia en un ejido de la Reserva de la Biosfera Calakmul, Campeche, México. Universidad y Ciencia 18: 11-28.

Galindo-Leal C (1999) La gran región de Calakmul, Campeche: Prioridades biológicas de conservación y propuesta de modificación de la Reserva de la Biósfera. Center for Conservation Biology, Stanford University. Stanford California. Reporte final a World Wildlife Fund-México. México. 40p.

García E (1988) Adaptación del sistema climatológico de Köepen a la República mexicana. México. 76p.

García-Licona JB, Esparza-Olguín LG, Martínez-Romero E (2014) Estructura y composición de la vegetación leñosa de selvas en diferentes estadios sucesionales en el ejido El Carmen II, Calakmul, México. Polibotánica 38: 1-26.

González-Valdivia N, Ochoa-Gaona S, Ferguson BG, Pozo C, Kampichler C, Pérez-Hernández I (2012) Análisis comparativo de la estructura, diversidad y composición de comunidades arbóreas de un paisaje agropecuario en Tabasco, México. Revista Mexicana de Biodiversidad 83: 83-99.

Hammer O, Harper DAT, Ryan PD (2001) PAST: Paleontological Statistics software package for education y analysis. Palaeontologia Electronica 4: 1-9.

Hutcheson K (1970) A test for comparing diversities based on the Shannon formula. Journal of Theoretical Biology 29: 151-154.

INEGI (2014) Datos de relieve continental: Continuo de elevaciones mexicano 3.0 (CEM 3.0) Área geoestadística estatal: Campeche. Instituto Nacional de Estadística y Geografía. http://www.inegi.org.mx/ geo/contenidos/datosrelieve/continental/descarga.aspx. Fecha de consulta 25 de enero de 2015. 
IUCN (2015) The IUCN Red List of Threatened Species Version 2015.1. Unión Internacional para la Conservación de la Naturaleza. http://www.iucnredlist.org. Fecha de consulta 1 de junio de 2015.

Kepleis P (2004) Forest extraction to theme parks: The modern history of land change. In: BL Turner II, Goghegan J and Foster D (ed) Integrated land change science and tropical deforestation in the Southern Yucatán. Oxford University Press, Oxford. pp: 39-59.

López OR (2009) Fisiología y ecología de comunidades arbóreas en hábitats inundables. Acta Biológica Panamensis 1: 68-86.

Magurran AE (2004) Measuring biological diversity. Blackwell Publishing, Oxford, UK. 256p.

Maldonado-Sánchez EA, Ochoa-Gaona S, Ramos-Reyes R, Guadarrama-Olivera MA, González-Valdivia N, de Jong BHJ (2016) La selva inundable de canacoite en Tabasco, México, una comunidad vegetal amenazada. Acta Botánica Mexicana 115: 75-101.

Marks CO, Nislow KH, Magilligan FJ (2014) Quantifying flooding regime in floodplain forests to guide river restoration. Elementa Science of the Anthropocene 2: 1-15.

Martínez E, Galindo-Leal C (2002) La vegetación de Calakmul, Campeche, México: clasificación, descripción y distribución. Boletín de la Sociedad Botánica de México 71: 7-32.

Martínez-Sánchez JL (2016) Comparación de la diversidad estructural de una selva alta perennifolia y una mediana subperennifolia en Tabasco, México. Madera y Bosques 22: 29-40.

Palacio-Aponte AG, Noriega-Trejo R, Zamora-Crescencio P (2002) Caracterización físico-geográfica del paisaje conocido como "bajos inundables". El caso del Área Natural Protegida Balamkín, Campeche. Investigaciones Geográficas, Boletín del instituto de Geografía, UNAM 49: 57-73.

SEMARNAT (2010) Norma Oficial Mexicana NOM-059-SEMARNAT-2010 Protección ambiental-especies nativas de México de flora y fauna silvestres-categorías de riesgo y especificaciones para su inclusión, exclusión o cambio-lista de especies en riesgo. Diario Oficial de la Federación, México. 78p.

Tun-Dzul FJ, Vester H, Durán-García R, Schmook B (2008) Estructura arbórea y variabilidad temporal del NDVI en los "bajos inundables" de la Península de Yucatán, México. Polibotánica 25: 69-90.

Vázquez-Negrín I, López-Pérez D, Montalvo-Urgel HE, Méndez Sánchez CA, Castillo Acosta O (2010) Estructura y composición florística de vegetación inundable en la División Académica de Ciencias Biológicas, Villahermosa, Tabasco. Kuxulkab’ Revista Divulgación 17: 21-30.

Villalobos-Zapata GJ, Mendoza-Vega J (2010) La biodiversidad en Campeche: Estudio de estado. Comisión Nacional para el Conocimiento y Uso de la Biodiversidad, Gobierno del Estado de Campeche, Universidad Autónoma de Campeche, El Colegio de la Frontera Sur. México. 730p.

Zamora-Crescencio P, García-Gil G, Flores-Guido JS, Javier-Ortíz J (2008) Estructura y composición florística de la selva mediana subcaducifolia en el sur del estado de Yucatán, México. Polibotánica 26: 39-66.

Zamora-Crescencio P, Gutiérrez-Báez C, Folan WJ, Domínguez-Carrasco M, Villegas P, Cabrera-Mis G, et al (2012) La vegetación leñosa del sitio arqueológico de Oxpemul, municipio de Calakmul, Campeche, México. Polibotánica 33: 131-150. 